\title{
Spectrally Compatible Iterative Water Filling
}

\author{
Jan Verlinden, ${ }^{1}$ Etienne Van den Bogaert, ${ }^{2}$ Tom Bostoen, ${ }^{1}$ Francesca Zanier, ${ }^{3}$ Marco Luise, ${ }^{4}$ \\ Raphael Cendrillon, ${ }^{5}$ and Marc Moonen ${ }^{6}$ \\ ${ }^{1}$ Access Networks Division, Alcatel, Francis Wellesplein 1, Antwerpen 2018, Belgium \\ ${ }^{2}$ Research \& Innovation Department, Alcatel, Francis Wellesplein 1, Antwerpen 2018, Belgium \\ ${ }^{3}$ Department of Telecommunication Engineering, University of Pisa, 56122 Pisa, Italy \\ ${ }^{4}$ Department of Information Engineering, University of Pisa, 56122 Pisa, Italy \\ ${ }^{5}$ School of Information Technology and Electrical Engineering, University of Queensland, Brisbane, QLD 4072, Australia \\ ${ }^{6}$ Department of Electrical Engineering, Katholieke Universiteit Leuven, Kasteelpark Arenberg 10, Leuven-Heverlee 3001, Belgium
}

Received 2 December 2004; Revised 1 July 2005; Accepted 12 July 2005

\begin{abstract}
Until now static spectrum management has ensured that DSL lines in the same cable are spectrally compatible under worstcase crosstalk conditions. Recently dynamic spectrum management (DSM) has been proposed aiming at an increased capacity utilization by adaptation of the transmit spectra of DSL lines to the actual crosstalk interference. In this paper, a new DSM method for downstream ADSL is derived from the well-known iterative water-filling (IWF) algorithm. The amount of boosting of this new DSM method is limited, such that it is spectrally compatible with ADSL. Hence it is referred to as spectrally compatible iterative water filling (SC-IWF). This paper focuses on the performance gains of SC-IWF. This method is an autonomous DSM method (DSM level 1) and it will be investigated together with two other DSM level-1 algorithms, under various noise conditions, namely, iterative water-filling algorithm, and flat power back-off (flat PBO).
\end{abstract}

Copyright () 2006 Hindawi Publishing Corporation. All rights reserved.

\section{INTRODUCTION}

More and more users today have broadband access to the Internet based on ADSL (asymmetric digital subscriber line) technology. However, still not all users can have this service, because they are located too far from the central office. People who have broadband access today prefer to have even higher data rates. DSM is a technique that tries to optimize the rates and reach for all users in a network. As such a telecom operator can offer broadband access to as many customers as possible and provide them with the optimal data rates.

Rate and reach for a certain DSL service are limited by crosstalk. Crosstalk is noise that comes from other DSL lines in the same binder. Due to electromagnetic coupling, the signal of one line indeed interferes with the signal of the other line.

DSM tries to optimize the spectrum that is used for a certain line, such that it does not allocate a higher power spectral density (PSD) than necessary to achieve the requested bit rate. For DSM, several algorithms exist. In this paper we will focus on DSM level 1, that is, autonomous algorithms that do not need coordination from a central agent. We will develop a new DSM method for downstream ADSL, referred to as spectrally compatible iterative water filling (SC-IWF); see also [1].

We will only apply DSM to the downstream direction (from the Internet to the user), because crosstalk coupling is negligible in the ADSL upstream band.

Whereas static spectrum management (SSM) will always consider the worst-case noise environment to design the maximum allowed PSD masks, DSM will use the actual measured noise and adapt its spectrum accordingly.

So, unlike the standardized test procedures for SSM [2], we will not focus only on the performance of a single line in a network with fixed noise. Instead, because of the dynamic PSD used by DSM, and because of the effect of crosstalk of one line on another line, we will consider multiple lines. Since we do not consider worst-case crosstalk, we will quantify the improvements in a real-life network.

The paper is organized as follows. In Section 2 the various DSM algorithms are described and SC-IWF is derived from IWF. In Section 3 rates-reach simulations are presented for a single line under test. In Section 4 a description of the network (cable plant) is given. In Section 5 the simulation results are presented. Finally, in Section 6 we present the conclusion of this paper. 


\section{DSM ALGORITHMS}

\subsection{Iterative water filling or full boosting}

\subsubsection{General properties}

A well-known autonomous DSM algorithm is iterative water filling (IWF); see also [3]. The behavior of IWF and the resulting PSDs has already been investigated in several other papers $[3,4]$. The main properties of IWF are the fact that only useful tones are switched on (all other tones are switched off) and that both boosting and power back-off are used. The amount of boosting is limited by the total power that can be transmitted.

For modems that use DMT (discrete multitone) modulation, like ADSL modems, one can prove that the PSDs obtained with IWF are almost flat [5]. This will be explained in the following paragraphs.

\subsubsection{PSDs of IWF}

IWF is obtained as a result of a bit rate maximization of multiple users, where each modem optimizes its own PSD under the assumption that the noise coming from other modems is constant. Since the other modems will also change their PSD, the noise measured by the first modem is not constant and it will also change its PSD. This results in an iteration of multiple modems applying the water-filling procedure, hence its name iterative water filling.

When using a Shannon capacity model for a DMT modem, the bit rate of the first modem (assuming a two-user system) is described as

$$
\begin{aligned}
R_{1} & =R_{S} \sum_{k} b_{k}^{1}, \\
b_{k}^{1} & =\log _{2}\left(1+\frac{\mathrm{SNR}_{1}(k)}{\Gamma_{1}}\right) \\
& =\log _{2}\left(1+\frac{h_{11}^{2}(k) S_{1}(k)}{\Gamma_{1}\left(N_{1}(k)+h_{12}^{2}(k) S_{2}(k)\right)}\right)
\end{aligned}
$$

with $R_{1}$ the bit rate of user $1, R_{S}$ the symbol rate, $k$ the tone index, $\operatorname{SNR}(k)$ the signal-to-noise ratio (SNR) on tone $k, \Gamma_{1}$ the SNR gap including noise margin and coding gain, $S_{i}(k)$ the transmit PSD of user $i$ on tone $k, h_{11}(k)$ the channel transfer function for user $1, h_{12}(k)$ the crosstalk channel transfer function from user 2 to user 1 , and $N_{1}(k)$ all the noises different from the crosstalk from user 2.

Maximizing the bit rate of both users leads to the following cost function, while taking into account the total power limitation of each user (constrained optimization problem):

$$
\begin{aligned}
J\left(S_{1}(k), S_{2}(k)\right)= & \sum_{k} \log _{2}\left(1+\frac{h_{11}^{2}(k) \cdot S_{1}(k)}{\Gamma_{1}\left(N_{1}(k)+h_{12}^{2}(k) \cdot S_{2}(k)\right)}\right) \\
& +\sum_{k} \log _{2}\left(1+\frac{h_{22}^{2}(k) \cdot S_{2}(k)}{\Gamma_{2}\left(N_{2}(k)+h_{21}^{2}(k) \cdot S_{1}(k)\right)}\right) \\
& +\lambda_{1} \cdot\left(P_{1}-\sum_{k} S_{1}(k)\right)+\lambda_{2} \cdot\left(P_{2}-\sum_{k} S_{2}(k)\right)
\end{aligned}
$$

with $P_{i}$ the maximum power of user $i$, and $\lambda_{i}$ the Lagrange multiplier of user $i$.

Assuming that the noise from other modems is constant (as highlighted in the first paragraph), one can fix the PSD in the crosstalk terms and formula (3) can be rewritten as

$$
\begin{aligned}
J\left(S_{1}(k),\right. & \left.S_{2}(k)\right) \\
= & \sum_{k} \log _{2}\left(1+\frac{h_{11}^{2}(k) \cdot S_{1}(k)}{\Gamma_{1} \tilde{N}_{1}(k)}\right) \\
& +\sum_{k} \log _{2}\left(1+\frac{h_{22}^{2}(k) \cdot S_{2}(k)}{\Gamma_{2} \tilde{N}_{2}(k)}\right) \\
& +\lambda_{1} \cdot\left(P_{1}-\sum_{k} S_{1}(k)\right)+\lambda_{2} \cdot\left(P_{2}-\sum_{k} S_{2}(k)\right)
\end{aligned}
$$

with $\tilde{N}_{1}(k)=N_{1}(k)+h_{12}^{2}(k) \cdot S_{2}^{\mathrm{fix}}(k)$ and $\tilde{N}_{2}(k)=N_{2}(k)+$ $h_{21}^{2}(k) \cdot S_{1}^{\mathrm{fix}}(k)$.

The cost function can now be decoupled in two independent cost functions for each user. This leads to the following solution for $S_{i}(k)$ :

$$
S_{i}(k)=\left[\frac{1}{\lambda_{i} \ln 2}-\frac{\Gamma_{i} \tilde{N}_{i}(k)}{h_{i i}^{2}(k)}\right]^{+}
$$

with $[x]^{+}=\max (0, x)$.

This procedure is applied in an iterative fashion until $S_{1}^{\mathrm{fix}}(k)=S_{1}(k)$ and $S_{2}^{\mathrm{fix}}(k)=S_{2}(k)[3]$.

This derivation assumes a rate adaptive system with a fixed amount of power. However, by controlling the power $P_{i}$, one can control the resulting bit rate. In that case, IWF is used in a power-adaptive perspective. In the remainder of this paper, IWF will be used in such a power-adaptive perspective, where the amount of power depends on the desired bit rate. In the simulations an additional iteration is used to determine the power that corresponds to a certain bit rate.

\subsubsection{The PSDs of IWF are almost flat}

Since the so-called water-filling level $\lambda_{i}$ is independent of the tone index $k$, the minimum and maximum values of $S_{i}(k)$ in formula (5) are determined by the second term:

$$
\frac{\Gamma_{i} \tilde{N}_{i}(k)}{h_{i i}^{2}(k)} \text {. }
$$

When this second term is very large, then the SNR for user $i$ will be smaller. Considering (2), one can observe that for DMT systems (like ADSL) on each tone $k$ an SNR equal to or larger than $\Gamma_{1}$ must be available, in order to load at least 1 bit, so the signal $S_{1}(k)$ needs to be at least as large as the term $(6)$.

This allows us to derive the minimum value for $S_{1}(k)$ :

$$
S_{1}^{\min }(k)=\frac{\Gamma_{1} \tilde{N}_{1}(k)}{h_{11}^{2}(k)}=\frac{1}{2 \lambda_{1} \ln 2} .
$$

When the term (6) is very small, we obtain the maximum value for $S_{1}(k)$ :

$$
S_{1}^{\max }(k)=\frac{1}{\lambda_{1} \ln 2}
$$


Comparing formulas (7) and (8) shows that the difference between $S_{1}^{\min }$ and $S_{1}^{\max }$ is only a factor 2 , which means that the difference in PSD level is only $3 \mathrm{~dB}$. This means that the transmit PSD can be approximated by a flat PSD for all the used tones. The small PSD ripple obtained when calculating the PSD with IWF does not have a significant effect on the bit rate. This has been observed in simulation, both for integer (using greedy algorithm) and continuous bit loadings, as long as the requirement of minimum 1 bit per tone is taken into account. ADSL modems in the field use integer bit loadings and will anyhow adapt their PSD within a range of $-2.5 \mathrm{~dB}$ to $+2.5 \mathrm{~dB}$ around the average PSD value (see also [6]). This mechanism is also known as the $g_{i}$ gain scaling mechanism. Note that this average PSD value can change in time over a much larger range than this $\pm 2.5 \mathrm{~dB}$. Hence no PSD shaping is required for IWF. The tones for which the SNR is not high enough are shut off. If the noise varies over time, the average PSD level will vary and the actual tones that are used (tones that are switched on) can change.

Basically, IWF consists in shutting off tones, boosting for long lines, and power back-off for short lines, always using a PSD that is almost flat.

During simulations always a flat PSD is used (both during the convergence period and at the end), ignoring the gain scaling factors and using a continuous bit loading with minimum 1 bit per tone.

\subsection{Spectrally compatible IWF}

\subsubsection{Spectrum management and compatibility}

The T1E1.4 Working Group of the T1 Committee (ANSI) has adopted a "Spectrum Management for Loop Transmission Systems" standard [7]. This standard provides spectrum management requirements and recommendations for the administration of services and technologies that use metallic subscriber loop cables. Spectrum management is the administration of the loop plant in a way that provides spectral compatibility for services and technologies that use pairs in the same cable.

In order to achieve spectral compatibility, the ingress energy that transfers into a loop pair from services and transmission system technologies on other pairs in the same cable must not cause an unacceptable degradation of performance of the DSL service of the loop under consideration. In addition, the egress energy from a particular loop pair must not transfer into other pairs in a manner that causes an unacceptable degradation in the performance of services and technologies on those pairs.

There are basically two ways to ensure spectral compatibility with the existing protected services: Method A and Method B. Method A consists of a series of fixed masks (management classes). In order to encourage innovation, Method $\mathrm{B}$ was proposed. This method provides a generic analytical method (instead of a fixed set of masks) for determining spectral compatibility. This method is more complicated than Method A, and consists in fact of a series of tests, which are done on a technology-by-technology basis. There are several "basis systems" defined, and for each one a specific test is performed.

\subsubsection{Spectral compatibility with ADSL}

In order to implement the Method B tests, a software tool (in Matlab) has been developed, following the specifications of [7]. In order to check the accuracy of the tool, the results have been compared with the Telcordia tool (Telcordia DSL Spectral Compatibility Computer; see [8]). The comparison has been made for an extensive number of cases, and there is a very good agreement between the results of both tools.

Alcatel's tool works as follows: a "new service" is defined corresponding to a PSD (power spectral density) in upstream and downstream. Then, by using a user interface one selects the basis systems (protected services) to be tested with. For spectral compatibility, throughout this contribution, only spectral compatibility with ADSL will be tested.

The reason why only ADSL is protected in the design of these new masks can be found in [9]. In this paper the probability of such a spectral incompatibility is calculated. This calculation is based on the fact that the worst-case crosstalk model only occurs for $1 \%$ of the lines, that there is only a problem if these systems are both installed in the same binder, and so forth. According to [9], it will probably be better (from a business point of view) to replace the few HDSL lines on which there is a spectral compatibility problem, with fiber, than to reduce the reach of ADSL and to reduce the number of customers that can be served (with ADSL) by imposing very strict limitations on the PSD of ADSL. This is why only spectral compatibility with ADSL is taken into account (since one can assume that ADSL will always be present in the binder, and as such always needs protection).

\subsubsection{Design of spectrally compatible PSD masks}

The original downstream ADSL mask is presented in Figure 1 with a solid line. It spans from $138 \mathrm{kHz}$ up to approximately $1.1 \mathrm{MHz}$, and has a level of $-40 \mathrm{dBm} / \mathrm{Hz}$.

Since the PSD of IWF can be approximated by a rectangular mask, only rectangular PSD masks are considered for the design of spectrally compatible PSD masks.

For this design, a two-dimensional search space is explored: in a first dimension the PSD level is increased (indicated with dash-dotted line), in a second dimension the maximum frequency of the mask is reduced (dashed line). The minimum frequency of the mask is kept fixed.

The selection of a one-dimensional set of new masks is based on two constraints: firstly there is the total transmit power constraint of ADSL that needs to be respected $(19.85 \mathrm{dBm})$ and secondly the masks need to be spectrally compatible with ADSL. Obviously, all the new masks will exceed the Method A spectral compatibility requirements (since they exceed the $-40 \mathrm{dBm}$ PSD limitation), and therefore Method B will be employed for ensuring spectral compatibility. 


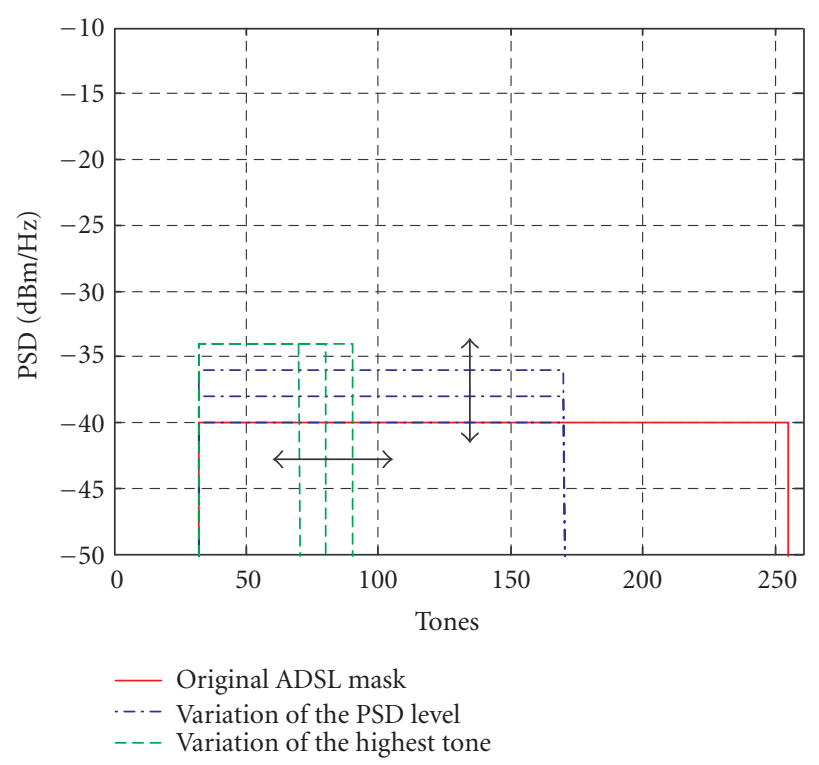

Figure 1: Search for spectrally compatible PSD masks.

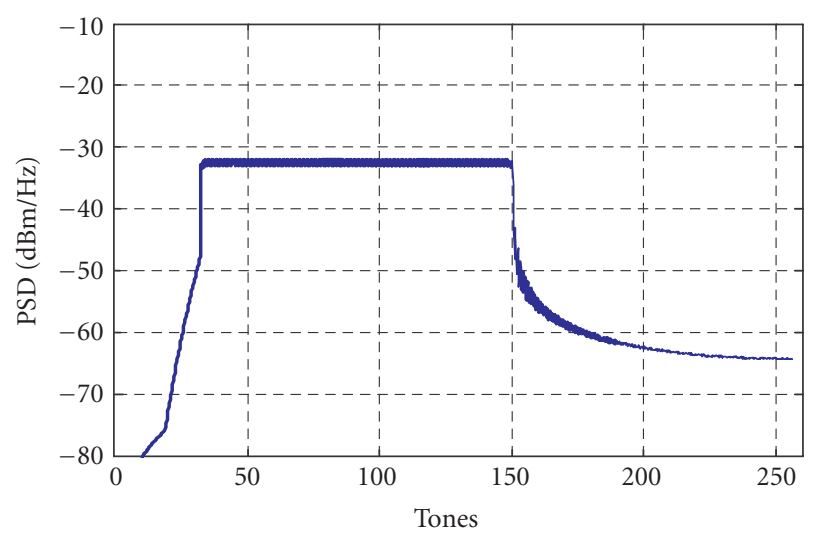

FIGURE 2: Example of a PSD, considered in the search algorithm, including sidelobes.

From all masks with the same maximum frequency, one mask will be selected. It will need to comply with the two constraints mentioned above and from all the masks fulfilling these constraints, the one with the highest level of boosting will be selected, since this mask will result in the highest bit rate.

All the above procedures were automated in a Matlab script, interfacing with Alcatel's Method B tool.

Sidelobes have been calculated for the PSD of the new system (see Figure 2). With these sidelobes the effect of energy spreading of the DMT modulation is taken into account. It is very important to model the energy on the tones that have been switched off, because the energy on such tones due to sidelobes can still give significant crosstalk for other

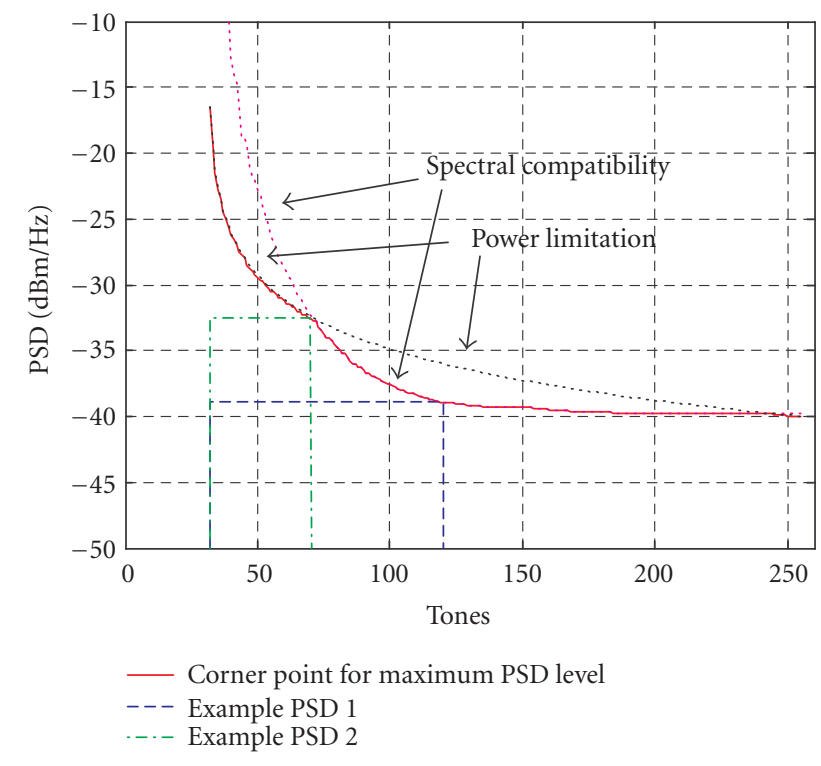

FIGURE 3: PSDs for spectrally compatible boosting (protecting ADSL services).

systems. The sidelobes have been calculated using matrix A from [10, Appendix IV].

The resulting spectrally compatible masks are presented in Figure 3. The corner point indicates for each mask (defined by a maximum frequency) the maximum transmit PSD level. Two examples (dashed and dash-dotted rectangles) indicate how the curve of corner points should be used.

In the figure, it is also indicated which constraint determines the PSD level: the maximum transmit power limitation or the spectral compatibility limitation. The maximum transmit power limitation is calculated by setting equal PSDs on all tones that are used. The spectral compatibility limitation is based on Method B of [7] as described before. This limitation indicates, for each tone $k$, the highest PSD that is still allowed, when using a rectangular mask that starts at the minimum tone (at $138 \mathrm{kHz}$ ) and ends at tone $k$.

\subsection{Flat power back off}

Since boosting is not allowed by all operators (T1.417 is an ANSI standard, only valid in North America), and since the spectral compatibility test is only protecting ADSL, also a flat PBO algorithm is used for comparison. This algorithm has the same behavior as IWF, but it will never exceed the maximum level of $-40 \mathrm{dBm} / \mathrm{Hz}$, which is currently defined for ADSL in [6].

\section{RATE-REACH SIMULATION RESULTS}

\subsection{Introduction}

We will first present the rate-reach curves for the various algorithms. For these rate-reach curves, only one modem under test is considered. Since the simulations consist only of 


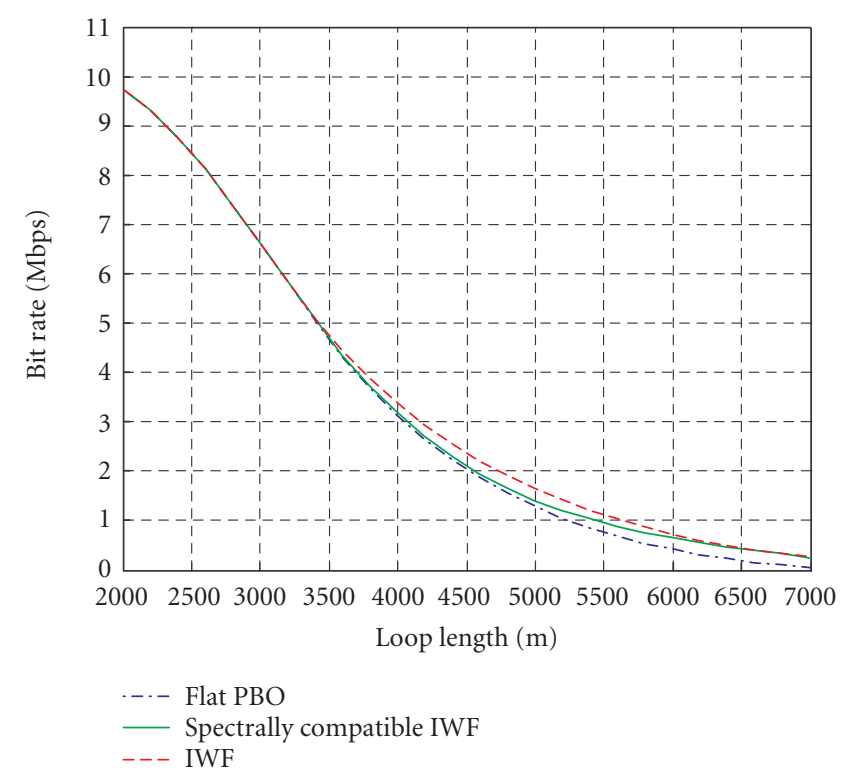

FIGURE 4: Rate-reach curve for the various DSM algorithms under AWGN of $-140 \mathrm{dBm} / \mathrm{Hz}$.

one modem under test, it will be easier to interpret the results. This is important because later on in this paper the results of DSM in a network with a large number of modems will be presented. In order to understand better how such a DSM modem works under fixed noise conditions, we need to investigate its behavior in a controlled noise environment.

\subsection{Rate-reach simulation results under AWGN noise conditions}

In Figure 4 one can see the rate-reach curve of the various DSM algorithms. DSM has been applied to one line only, in an environment with AWGN at $-140 \mathrm{dBm} / \mathrm{Hz}$.

One can clearly see that there is only a difference between IWF and flat PBO on the longer loops. On these long loops, not all tones are used any more and so IWF will reuse the power from these tones to apply boosting. This boosting will result in the improved bit rates.

The SC-IWF algorithm first behaves as flat PBO on the medium length loops, and coincides with IWF for the long loops. This can be explained by closely looking at Figure 3. There one can observe that when a large number of tones are used, SC-IWF is limited by the spectral compatibility constraint. But as more tones become unavailable (due to the longer loop length and corresponding channel attenuation), SC-IWF will be only limited by the power constraint and will in fact have the same PSD as full IWF. Hence, for the longer loops, IWF and SC-IWF will have the same PSD and corresponding bit rate.

\subsection{Rate-reach simulation results under ETSI-FB noise conditions}

In Figure 5 one can see the rate-reach curve of the various DSM algorithms under ETSI-FB noise (see [10-12]), consisting of 10 ISDN (=ISDN-BRA, 2B1Q), 4 HDSL, 15 SHDSL (=SDSL) disturbers, and 15 legacy ADSL disturbers. The bit rates are much lower than under AWGN $-140 \mathrm{dBm} / \mathrm{Hz}$ noise.

In Figure 5(a), the mask selection of SC-IWF is based on the "maximum tone." This means that the algorithm will search for the highest tone that can still be used and will select the corresponding mask from Figure 3, using this maximum tone as the corner point.

In Figure 5(b), the mask selection is based on the maximum bit rate. This means that in the receiver (which determines the selection of the mask) the bit rate for all masks from Figure 3 is calculated and the mask that results in the highest bit rate is selected. Since the ETSI-FB noise is very shaped, and will cause a gap of tones (tones which cannot be used), optimal mask selection, based on maximization of the bit rate, is indeed seen to lead to higher bit rates. The drop in bit rate, observed in Figure 5(a), can be explained as follows. When there are only one few tones available after the gap of useless tones, then it makes sense to switch off also these tones, and use a higher PSD on the tones before the gap.

\section{NETWORK WIDE APPLICATION}

\subsection{Realistic model of the network}

An important difference between the results in this paper and other simulation results (in standards or other papers) is the fact that we use a realistic network topology in which we investigate the gains of DSM. Other papers usually work with either fixed noise or only a limited number of DSM modems (e.g., two) on a fixed binder setup.

However, the main gain for DSM comes from the fact that DSM modems adapt their PSD according to the loop and noise characteristics. For example, a modem on a short line will apply power back-off to reduce the noise for other users, whereas a modem on a long line will apply boosting in order to fully exploit the few tones that are still useful. Because of these changing PSDs, the noise from DSM modems is in fact not fixed and depends very much on the actual topology. So in order to investigate the gains of DSM in case it would be deployed on a large scale (more than 2 modems in a binder), it is important to have a realistic model of the network.

We use a network model of an average North American network and an average European network. The loop length distributions from the central office $(\mathrm{CO})$ for both of them are presented in Figure 6. In the North American network the loops are longer on the average. For the simulations a wire gauge of 26 AWG is used.

\subsection{Noise environment}

\subsubsection{Introduction}

The performance on a DSL line is limited mainly by the loop length and the noise environment. So the actual choice of the noise sources is rather important. In this analysis only the noise from crosstalk is considered. 


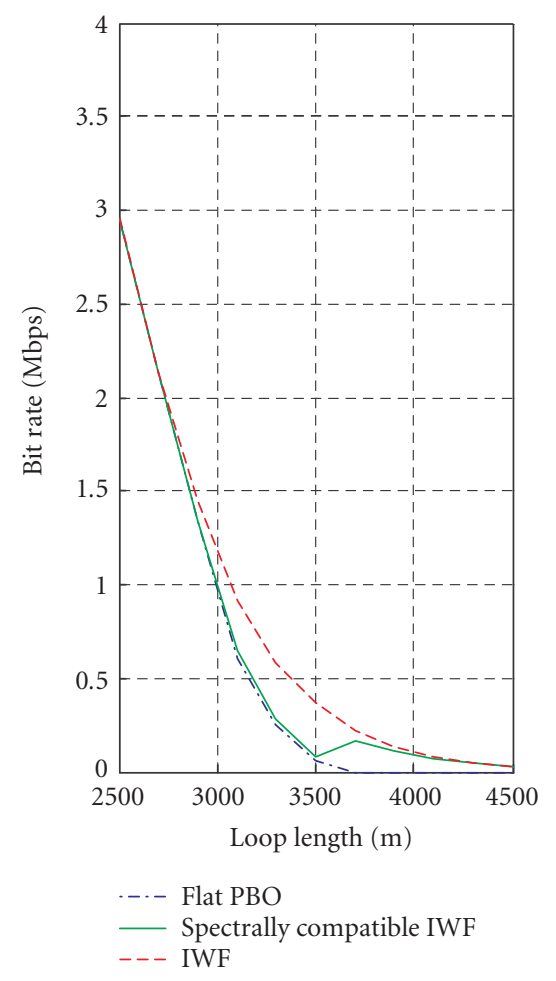

(a)

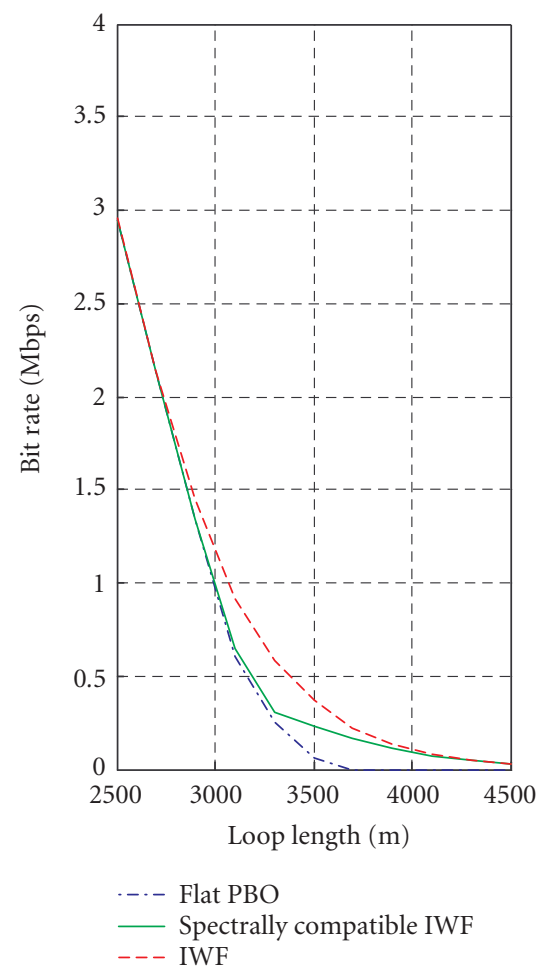

(b)

FIGURE 5: Rate-reach curve for the various DSM algorithms under ETSI-FB noise.

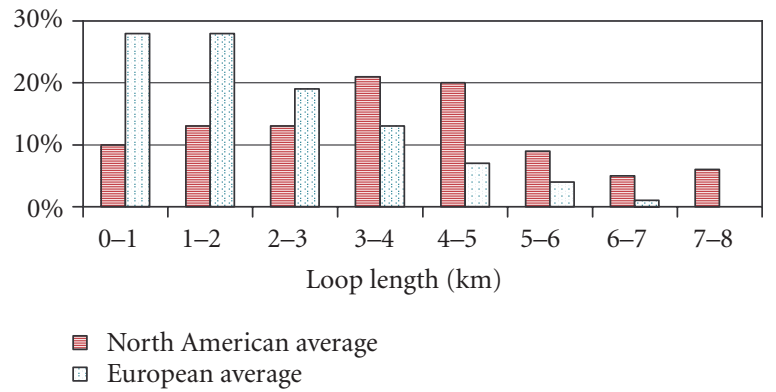

FIgURE 6: Loop length distribution (of homes extending out of CO).

This crosstalk noise is divided into two main categories: near-end crosstalk (NEXT) and far-end crosstalk (FEXT). NEXT is noise coming from a modem that is located at the same end of the line: if the victim modem is located in the central office (CO), then also the disturber is located in the CO. FEXT is noise coming from a modem located at the other end of the line: if the victim modem is located at the $\mathrm{CO}$, then the disturber is located at the customer premises (CPE).

\subsubsection{Noise sources}

All simulations have been performed with 16 ADSL modems that apply DSM. This number originates from the ETSI-FB model. As already mentioned in Section 3.3, there are 10 ISDN lines, 4 HDSL lines, 15 SHDSL lines, 15 ADSL lines, and 1 line under test in this model. So, in total there are 16 ADSL modems in this ETSI-FB model.

For the tests we will define the following noise scenarios: (a) AWGN noise at $-140 \mathrm{dBm} / \mathrm{Hz}$, (b) pure self-crosstalk noise (16 legacy ADSL lines, not applying DSM) derived from ETSI-FD, and (c) the noise from ETSI-FB (10 ISDN, 4 HDSL, 15 SHDSL).

In fact, noise scenario (a) corresponds to a case with all lines in a binder doing DSM, scenario (b) corresponds to $50 \%$ of the lines applying DSM, and for scenario (c) the effect of alien crosstalkers on DSM is investigated.

\subsection{Bandwidth tier bit rates}

\subsubsection{Bit rates in function of services}

Because of loop attenuation, the maximum achievable bit rate on a line will decrease with the loop length. So, customers on short loops can have a higher bit rate than customers on a long loop. However, since operators want to offer a package of services like high-speed Internet (HSI), voice over IP (VoIP), and video on demand, they will limit the achievable bit rate to the bit rate that is actually required for a certain package of services.

For the simulations, the following profiles have been chosen (see also Figure 7):

(i) Tier 0: $256 \mathrm{kbps}$ for broadband Internet connectivity, 


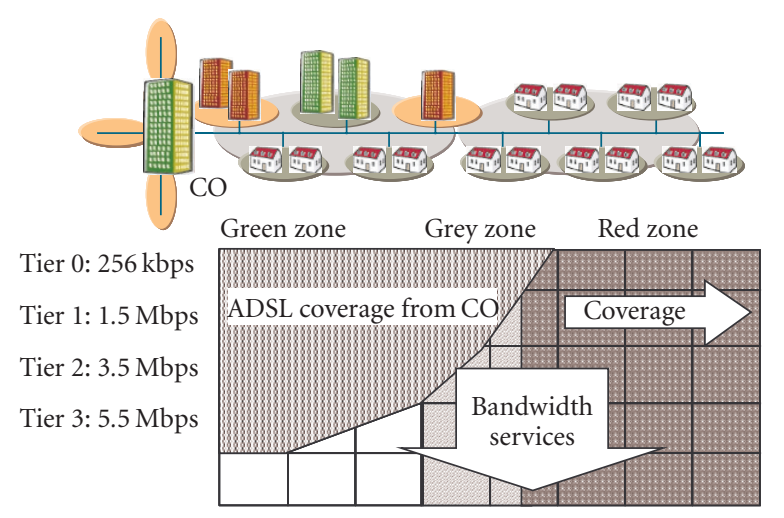

Figure 7: Tier bit rates.

(ii) Tier 1: 1.5 Mbps for high-speed Internet connectivity,

(iii) Tier 2: $3.5 \mathrm{Mbps}$ for high-speed Internet connectivity and one $2 \mathrm{Mbps}$ video channel,

(iv) Tier 3: $5.5 \mathrm{Mbps}$ for high-speed Internet, and two 2 Mbps video channels.

Unlike an operator, who installs a profile depending on the subscription of the customer, we will always enable the highest possible profile on a line.

\subsubsection{Effect of tier bit rates for DSM}

The profile that is selected for a line is always lower than the maximum achievable bit rate (otherwise this profile would not be possible). The amount of excess bit rate is converted by DSM into power back-off, such that the crosstalk for other users is reduced.

\section{SIMULATION RESULTS}

\subsection{Introduction}

In order to obtain results for a particular network, several simulations have been performed for this network. For each scenario, 30 binders have been generated and the downstream bit rates for all modems in a binder have been analyzed statistically for these 30 binders.

Each binder consists of loops that have been selected randomly from the loop distribution. Per binder, there are 16 ADSL modems applying DSM and several other lines acting as noise sources (the amount and type of disturbers depend on the noise scenario). So, per noise scenario, the downstream bit rates of 480 ADSL modems applying DSM are analyzed.

Reach curves are used to evaluate the performance gains of DSM.

\subsection{Reach curves per tier bit rate for the various DSM algorithms}

In this section, the results from the simulations with the noises from Section 4 are presented. First the results for the

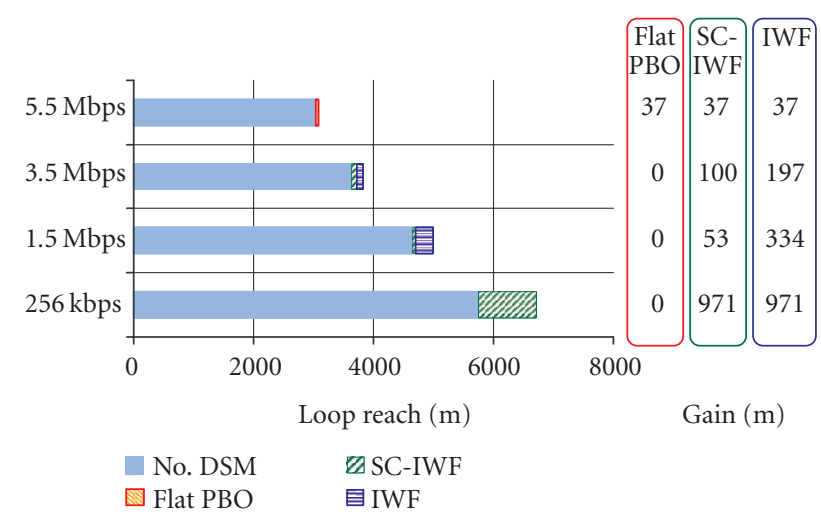

FIgURE 8: Reach improvement by applying DSM for 16 ADSL modems in Europe with AWGN $-140 \mathrm{dBm} / \mathrm{Hz}$.

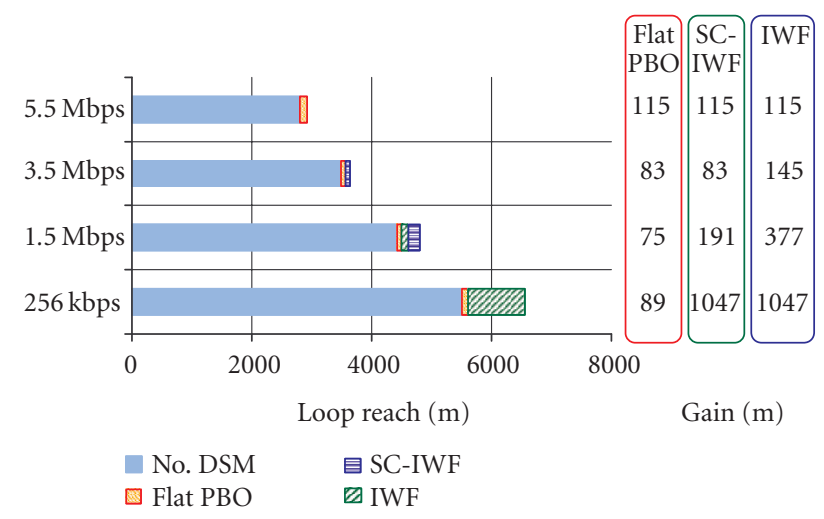

FIGURE 9: Reach improvement by applying DSM for 16 ADSL modems in Europe with 16 ADSL crosstalkers.

European network are presented (Figures 8, 9, and 10), and then the results for the North American network are given (Figures 11, 12, and 13).

As can be seen in Figures 8 and 9, it turns out that adding 16 ADSL self-crosstalkers is not really a problem for the ADSL lines under test. The loop reach and the gains for white noise and for ADSL noise are comparable.

For the $1.5 \mathrm{Mbps}$ service, there is only a substantial gain for IWF. Considering the average loop attenuation and the requested bit rate for this service, we know that still a lot of tones are useful. From Figure 3 we know that in this case, the boosting of the SC-IWF is very limited, and therefore there are almost no gains any more for this type of DSM.

For the $256 \mathrm{kbps}$ service, only a few tones are active on very long loops, so the amount of boosting for IWF and for SC-IWF is the same. Also the gains are identical.

The noise mix from ETSI-FB (Figure 10) has a big impact on the loop reach and it also reduces the gains, especially for the SC-IWF, using the $256 \mathrm{kbps}$ service. Due to the high noise level, the reach is reduced very much. For these shorter loops, the higher tones are still useful. As such, the amount of boosting that is allowed for the SC-IWF is reduced and also the gains. 


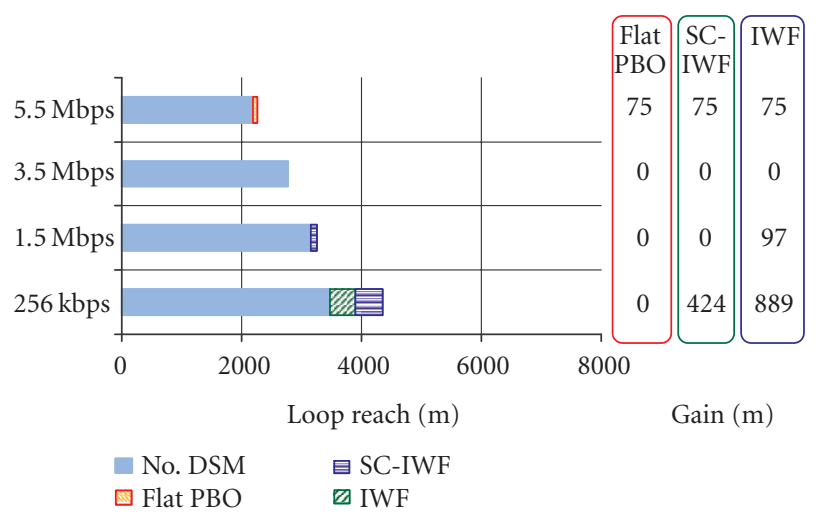

FIGURE 10: Reach improvement by applying DSM for 16 ADSL modems in Europe with 10 ISDN, 4 HDSL, and 15 SHDSL crosstalkers.

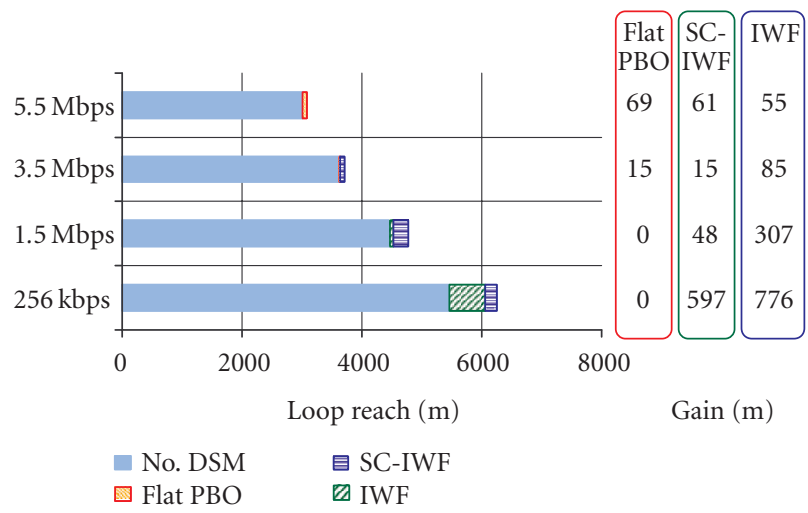

FIgURE 11: Reach improvement by applying DSM for 16 ADSL modems in North America with AWGN - $140 \mathrm{dBm} / \mathrm{Hz}$.

Comparing the results for the North American network (Figures 11, 12, and 13) with the results for the European network (Figures 8, 9, and 10), one can see that in general the DSM reach improvements for the European network are larger. The reason is that there are more short loops in the European network that can apply power back-off (reduction of crosstalk). The general trends however are the same, even though in both networks the loop distributions are very different.

\subsection{Comparing the effect of the various noises for the reach performance}

In the previous section, the noises from ETSI-FB (ISDN, HDSL, and SHDSL) were treated as a whole. In this section the contribution of the individual components of ETSI-FB is briefly investigated.

In Figure 14, one can see the performance gains of IWF on the North American network for each contribution of the ETSI-FB noises separately. The ISDN noise is clearly the least important noise source as the reach with ISDN noise only is much larger than with the other noise components.

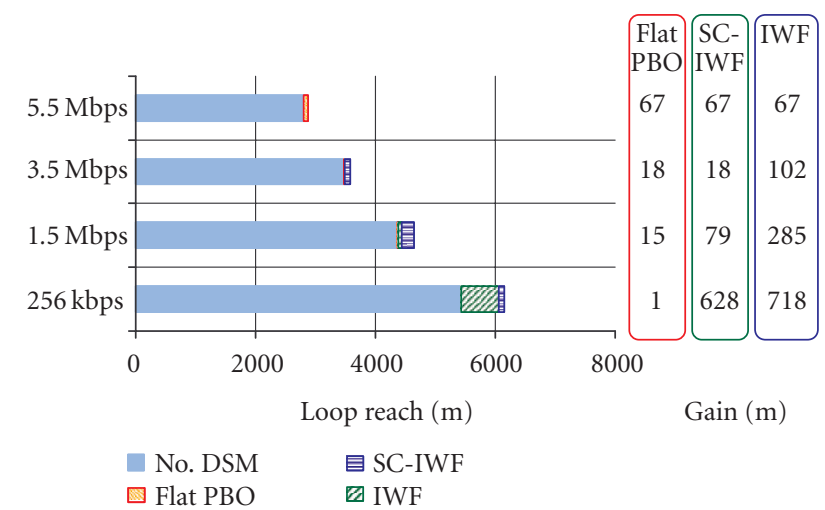

FIGURE 12: Reach improvement by applying DSM for 16 ADSL modems in North America with 16 ADSL crosstalkers.

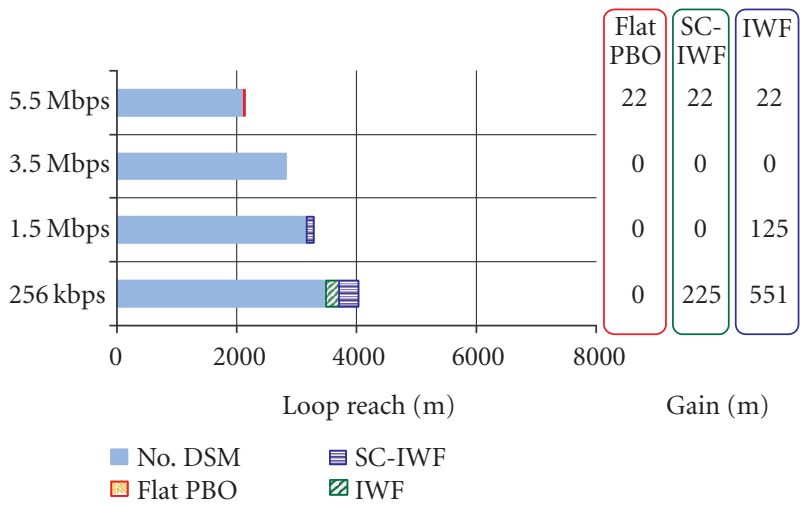

FIGURE 13: Reach improvement by applying DSM for 16 ADSL modems in North America with 10 ISDN, 4 HDSL, and 15 SHDSL crosstalkers.

HDSL and SHDSL (in the respective quantities of 4 and 15) have comparable impact on the reach. Both noises generate a lot of NEXT, which becomes the dominant noise source.

More detailed analysis shows that a single SHDSL noise source causes less degradation than a single HDSL noise source. However, the ETSI-FB model assumes that there will be more SHDSL disturbers in a network than HDSL disturbers.

It also turns out that in case there would be only 1 HDSL disturber instead of 4 , the reach (averaged over all services) is approximately $250 \mathrm{~m}$ larger. In case there would be only 1 SHDSL disturber instead of 15, the reach would be $300 \mathrm{~m}$ larger. This means that by reducing the number of HDSL and SHDSL disturbers in a network, the reach can also be improved.

\section{CONCLUSION}

In this paper we have investigated and compared existing algorithms for DSM level 1 with a new so-called spectrally compatible iterative water-filling (SC-IWF) algorithm. The 


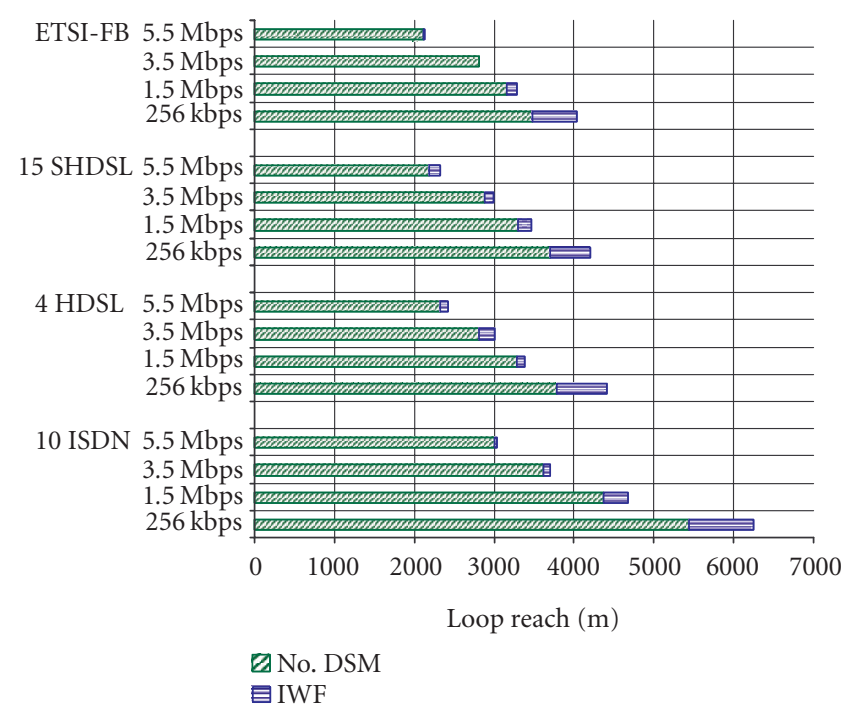

FIGURE 14: DSM performance for noise contributions of ETSI-FB.

results are based on statistical simulations in average European and North American networks. Different noise sources have been used as disturbers for the DSM lines under test.

When comparing the various algorithms it turns out that flat power back off (flat $\mathrm{PBO}$ ) only gives a small gain. This gain is most significant on the high bit rate profiles in a network with a large number of short lines.

SC-IWF (spectrally compatible with ADSL) gives a lot more gain and can perform almost as good as IWF, when working with the low bit rate profile. Gains are most significant for the long loops.

The gains of SC-IWF are reduced however in case a lot of tones are needed, either because of the profile (high bit rate) or because of the limited reach (high noise from NEXT disturbers). NEXT interference from HDSL and SHDSL in particular is found to have a large impact on the performance of ADSL, both with and without DSM.

\section{REFERENCES}

[1] R. Suciu, E. Van den Bogaert, J. Verlinden, and T. Bostoen, "Insuring spectral compatibility of Iterative Water-Filling," in Proceedings of 12th European Signal Processing Conference (EUSIPCO '04), pp. 1209-1212, Vienna, Austria, September 2004.

[2] ITU-T Recommendation G.996.1, Test procedures for digital subscriber line (DSL) transceivers, March 2003.

[3] W. Yu, G. Ginis, and J. M. Cioff, "Distributed multiuser power control for digital subscriber lines," IEEE Journal on Selected Areas in Communications, vol. 20, no. 5, pp. 1105-1115, 2002.

[4] R. Cendrillon, W. Yu, J. Verlinden, T. Bostoen, and M. Moonen, "Optimal multi-user spectrum management for digital subscriber lines," in IEEE International Communications conference (ICC '04), Paris, France, 2004.

[5] J. Verlinden, The target PSD obtained with Iterative WaterFilling is almost flat, ANSI Contribution. T1E1.4/2003-295, 2003.

[6] ITU-T Recommendation G.992.1, Asymmetric Digital Subscriber Line (ADSL) Transceivers, December 2003.
[7] ANSI Standard T1.417-2003, Spectrum Management for Loop Transmission Systems, December 2003.

[8] The Telcordia DSL Spectral Compatibility Computer, http:// net3.argreenhouse.com:8080/dsl-test/index.htm.

[9] J. M. Cioffi, Incentive-based spectrum management, ANSI T1E1.4/2004-480R2.

[10] ITU-T Recommendation G.992.5, Asymmetric Digital Subscriber Line (ADSL) Transceivers-Extended Bandwidth ADSL2 (ADSL2plus), January 2005.

[11] ETSI TS 101388 V1.3.1, ADSL: European Specific Requirements, section 5.3.4, May 2002.

[12] ETSI STC TM6(98)10, Laboratory Performance Tests for xDSL Systems, section B2, February 2001.

Jan Verlinden received a degree in electrical engineering in 2000 from the KULeuven, Belgium. He is currently a Member of the DSL Experts Team of the Access Network Division in Antwerp, Belgium. He joined the Research and Innovation Division of Alcatel in September 2000, where he focussed on echo canceller techniques. From 2002 on, he has focussed on DSM. As such he participated in the VDSL Olympics by in-

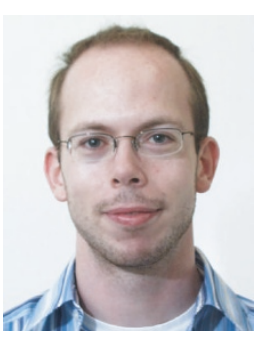
troducing DSM into the VDSL prototype. Within the DSL Experts Team, he is currently studying emerging DSL physical layer technologies. He also contributes to ANSI NIPP-NAI standardization.

Etienne Van den Bogaert is a DSL Research Engineer at the Research \& Innovation Department in Antwerp, Belgium. In this function, he is investigating nextgeneration DSL technologies and their applications. His current main research interest is dynamic spectrum management (DSM): algorithms, impact on performance and stability, and practical implementation.

Tom Bostoen received the M.S. degree in physical engineering from Ghent University, Ghent, Belgium, in 1998. Tom Bostoen is currently Product Manager of the 5530 Network Analyzer at the Access Networks Division of Alcatel in Antwerp, Belgium. In his previous function he was a Project Manager of the DSL physical layer research project at the Research \& Innovation Department. Before that, he studied single-

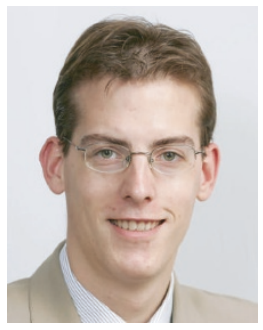
ended line testing (SELT) as a Research Engineer in the same department and contributed to ITU G.selt standardization.

Francesca Zanier received the degree in telecommunication engineering from Pisa University, Italy, in 2004. She joined the Research and Innovation Division of Alcatel, Antwerp, Belgium, in 2003 for her thesis's project on DSM. After winning the "Piaggio Talent Recruitment Project 2004", she worked one year in the Planning and Control Division of Piaggio Pontedera, Pisa (Italy). Recently she has joined the Department of Information Engineering as a PhD student at the University of Pisa (Italy). Her research interest is on digital signal processing. 
Marco Luise is a Full Professor of telecommunications at the University of Pisa, Italy. After receiving his M.S. and Ph.D. degrees in electronic engineering from the University of Pisa, he was a Research Fellow of the European Space Agency (ESA) at ESTEC Noordwijk, the Netherlands, and a Research Scientist of the Italian National Research Council (CNR), at the CSMDR Pisa. Professor Luise cochaired four editions of

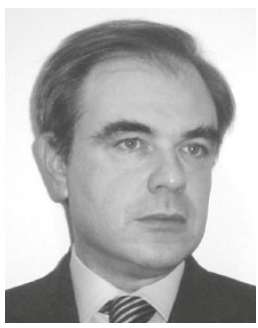
the Tyrrhenian International Workshop on Digital Communications, and in 1998 was the General Chairman of the URSI Symposium ISSSE'98. He has been the Technical Cochairman of the 7th International Workshop on Digital Signal Processing Techniques for Space Communications and of the Conference European Wireless 2002. He will be the General Chairman of EUSIPCO 2006 in Florence. A Senior Member of the IEEE, Professor Luise served as Editor for synchronization of the IEEE transactions on communications, and Editor for communications theory of the European transactions on telecommunications. He has authored more than 100 publications on international journals and contributions to major international conferences, and holds a few international patents. His main research interests lie in the area of wireless communications, with particular emphasis on CDMA/multicarrier signals and satellite communications and positioning.

Raphael Cendrillon was born in Melbourne, Australia, in 1978. He received the Electrical Engineering degree (first-class honors) from the University of Queensland, Australia, in 1999, and the Ph.D. degree in electrical engineering from the Katholieke Universiteit Leuven, Belgium, in 2004. His $\mathrm{Ph} . \mathrm{D}$. degree was awarded summa cum laude with congratulations of the jury, an honor given to the top 5\% of Ph.D. gradu-

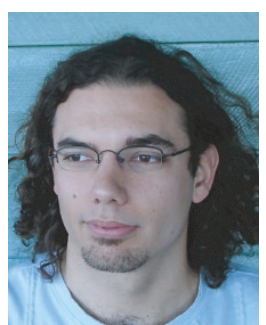
ates. His research focuses on the application of multiuser communication theory to xDSL. In 2002 he was a Visiting Scholar at the Information Systems Laboratory, Stanford University, with Professor John Cioffi. Since 2005 Dr. Cendrillon has been a Postdoctoral Research Fellow at the University of Queensland, Australia. His work in XDSL is done in close collaboration with Alcatel Research and Innovation, Belgium, for which he was awarded the Alcatel Bell Scientific Prize in 2004. He was also the recipient of an IEEE Travel Grant in 2003 and 2004, and the KULeuven Bursary for Advanced Foreign Scholars in 2004.

Marc Moonen received the Electrical Engineering degree and the Ph.D. degree in applied sciences from Katholieke Universiteit Leuven, Leuven, Belgium, in 1986 and 1990, respectively. Since 2004 he is a Full Professor at the Electrical Engineering Department of Katholieke Universiteit Leuven, where he is currently heading a research team of 16 Ph.D. candidates and Postdocs, working in the area

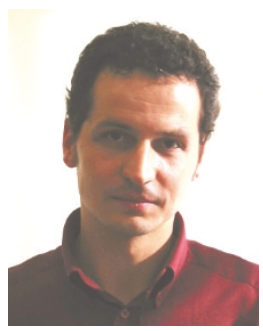
of signal processing for digital communications, wireless communications, DSL, and audio signal processing. He received the 1994 KULeuven Research Council Award, the 1997 Alcatel Bell (Belgium) Award (with Piet Vandaele), the 2004 Alcatel Bell (Belgium) Award (with Raphael Cendrillon), and was a 1997 "Laureate of the Belgium Royal Academy of Science." He was Chairman of the IEEE Benelux Signal Processing Chapter (1998-
2002), and is currently a EURASIP AdCom Member (European Association for Signal, Speech, and Image Processing) since 2000. He has been a Member of the Editorial Board of IEEE Transactions on Circuits and Systems II (2002-2003). He is currently Editor-inChief for the EURASIP Journal on Applied Signal Processing since 2003, and a Member of the Editorial Board of Integration, the VLSI Journal, EURASIP Journal on Wireless Communications and Networking, and IEEE Signal Processing Magazine. 